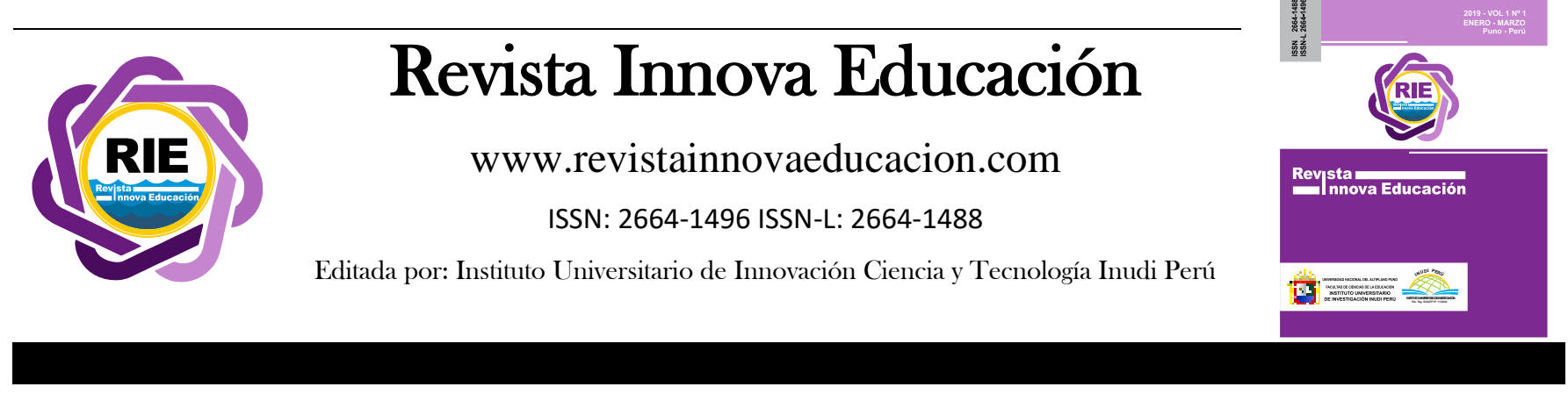

\title{
Externalidades del gasto público destinado a la educación en el crecimiento económico
}

\author{
Externalities of public spending for education in economic growth \\ Carmen Barrientos ${ }^{1}$ \\ Universidad Nur, Santa Cruz - Santa Cruz, Bolivia \\ https://orcid.org/0000-0001-6061-5283
}

DOI: https://doi.org/10.35622/j.rie.2020.01.007

Recibido el 18/12/2019/ Aceptado el 25/01/2020 Publicado el 31/01/2020

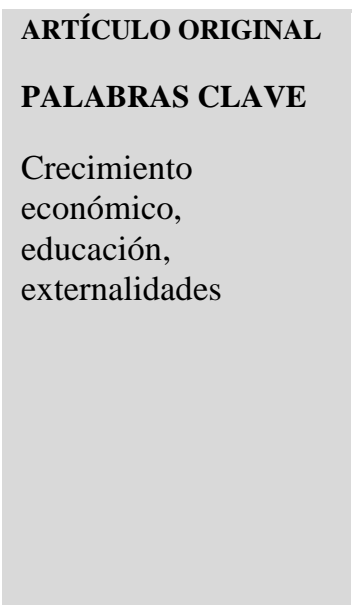

\section{KEYWORDS}

Economic growth, education, externalities
Al ser considerado el crecimiento económico como una medida del bienestar de la población, se constituye en uno de los objetivos básicos de la política económica; por lo tanto, el estudio de los factores o causas que la originan, ha sido, y continúa siendo, uno de los temas relevantes en la discusión económica. Lo expuesto dio origen al interés de determinar si el gasto público destinado a la educación generó una externalidad positiva o negativa en el crecimiento económico de los países de la región andina en el periodo 2000-2015. El estudio se realizó bajo el enfoque cuantitativo, específicamente por medio del desarrollo de un modelo econométrico. Los principales resultados evidencian que en el periodo de estudio, los países de la región andina se desarrollaron bajo contextos y políticas similares, donde las características y decisiones propias de cada país respecto al gasto público, si bien los diferenciaron, a su vez permitieron identificar una reacción conjunta a nivel de la región, en cuanto a los resultados de las políticas sociales, concluyendo que el gasto público destinado a la educación llegó a generar externalidades positivas en el crecimiento económico de la región andina.

\footnotetext{
${ }^{1}$ Correspondencia: armen.barrientos@nur.edu
}

When economic growth is considered as a measure of the welfare of the population, it constitutes one of the basic objectives of economic policy; Therefore, the study of the factors or causes that originate it has been, and continues to be, one of the relevant topics in the economic discussion. The foregoing gave rise to the interest of determining whether public spending for education generated a positive or negative externality in the economic growth of the countries of the Andean region in the period 2000-2015. The study was conducted under the quantitative approach, specifically through the development of an econometric model. The main results show that in the study period, the countries of the Andean region developed under similar contexts and policies, where the characteristics and decisions of each country regarding public spending, although they differentiated them, in turn allowed to identify a joint reaction at the level of the region, regarding the results of social policies, concluding that public spending for education came to generate positive externalities in the economic growth of the Andean region. 


\section{INTRODUCCIÓN}

Existe una vasta literatura teórica y empírica relacionada con la teoría de crecimiento económico y los factores que lo determinan; los estudios difieren desde el punto de vista de análisis, así como desde la concepción del contexto social, histórico, político, institucional, geográfico entre otros; sin embargo, a pesar del gran bagaje bibliográfico, no existe consenso de los factores que ocasionan un mayor o menor crecimiento.

En ese contexto, los trabajos de investigación de Solow (1957), Romer (1986), Barro (1990), Mankiw, Romer y Weil (1992), Kaufmann y Kraay (2002), Sala-i-Martin (2002), Glaeser, LaPorta, Lopes y Shleifer (2004), Acemoglu, Johnson y Robinson (2004), Rajan y Zingales (2006) y Ravallion (2014) permitieron identificar tres periodos de estudio: el primero, desde los años 50`s hasta mediados de los 80`s, donde se consideró a la acumulación de capital físico acompañada del desarrollo tecnológico como el factores predominantes en el crecimiento económico; posteriormente, el segundo periodo, entre los años 80’s y mediados del 2000, los estudios concluyeron que la acumulación del capital humano y del capital público son factores adicionales que promueven el crecimiento económico en los países y, finalmente el tercer periodo, desde mediados del año 2000 hasta la fecha, donde se continua estudiando al capital humano como variable fundamental del crecimiento económico pero desde un enfoque social e institucional; a partir del estudio de la implementación de políticas sociales que favorecen el bienestar individual y disminuyen la desigualdad social.

Respecto al capital humano y capital público, diferentes investigaciones dan cuenta de la necesidad de estudiar el efecto que ocasionan en el crecimiento económico. Es así, que se cuenta con aportes de Lucas (1988), argumentando que el proceso de adquisición de capital humano se da a través de dos fuentes: la educación formal propiamente dicha y el aprendizaje por la práctica. Asimismo, que las diferencias entre las tasas de crecimiento de los países son atribuibles a la tasa a la cual las economías acumulan capital humano en el tiempo. Por su parte, Becker (1995) señala que sistemas educacionales más exigentes, culturas que valoran más la educación preparan más y mejor a las personas para desenvolverse de manera más productiva en el mercado laboral, contribuyendo de esta manera en el crecimiento económico.

Una característica común que se encuentra en los aportes de Lucas y Becker es que, a través de una mejor educación se logra una mayor productividad en los trabajadores; que si se analiza desde el ámbito de las externalidades ${ }^{2}$, se puede concluir señalando que: "las externalidades

\footnotetext{
${ }^{2}$ La externalidad se da cuando se producen efectos indirectos de las actividades de una persona en el bienestar de otra y se manifiesta de dos formas, como una externalidad positiva, cuando el efecto indirecto beneficia en el bienestar de la otra persona, y negativa cuando el efecto es contrario.
} 
positivas o beneficios colectivos que genera la educación involucran, en particular, la capacidad de innovación y de competitividad de los trabajadores, impactando favorablemente en el crecimiento económico de un país”.

También se cuentan con trabajos que relacionan la salud con la educación y con el crecimiento económico, los mismos son analizados desde dos enfoques, el primero, aborda un sentido de dependencia, entre mayor ingreso de los individuos - buen estado de salud - generador de mayor crecimiento económico y, el segundo plantea el vínculo entre políticas de salud orientadas a la atención primaria - buen estado de salud - generador de mayor crecimiento económico (Aghion y Howitt, 1998). En esa dirección, Mogollón (2009:7) sostiene que, la salud de la población se encuentra estrechamente vinculada con la educación, la acumulación de capital humano y la productividad de la fuerza laboral y, es a través de ese vínculo que se logra un mayor crecimiento económico. En palabras del autor: “Trabajadores más saludables son más productivos, una mayor esperanza de vida brinda mayores incentivos para que las personas, se eduquen más, con lo cual se incrementa el stock de capital humano, los cuidados de salud en la niñez mejoran la capacidad de aprendizaje de las personas, generando un mayor stock de capital humano efectivo, los cuidados en la niñez hacen a las personas más creativas, innovadoras y con mayores habilidades para adaptarse a entornos cambiantes debido al rápido cambio tecnológico, la focalización de los cuidados de salud en las personas menos favorecidas de la sociedad contribuye a disminuir el grado de desigualdad social".

En ese contexto, Valdés y Espina (2011) incorporan a los bienes públicos como eje orientador de la política social y que forma parte de las políticas públicas. Según expresan los autores, la política social está referida a la entrega de bienes públicos o semipúblicos, donde no existen mercados completos o existen problemas redistributivos complejos. Al respecto, Katz citado por Dick (2010) señala que la provisión de los bienes públicos debe estar a cargo del Estado en respuesta a necesidades básicas fundamentales y ante la falta de provisión por parte del mercado o de la sociedad. El autor hace referencia principalmente a la salud pública, educación, condiciones de trabajo, vivienda, previsión social, defensa nacional y seguridad interior.

Lo expuesto, permitió formular la siguiente pregunta de investigación: ¿Qué tipo de externalidad generó el gasto público destinado a la educación en el crecimiento económico de los países que conforman la región andina en el periodo 2000-2015? De la revisión teórica efectuada se planteó como hipótesis del trabajo: El gasto público destinado a la educación generó un beneficio colectivo o externalidad positiva en el crecimiento económico de los países que conforman la región andina en el periodo 2000-2015. 
Finalmente, el trabajo tuvo como propósito determinar: si el gasto público destinado a la educación generó una externalidad positiva o negativa en el crecimiento económico de los países de la región andina en el periodo 2000-2015.

\section{MÉTODO Y MATERIALES}

En cuanto al método, la investigación se apoyó en el método de investigación teórico "hipotético deductivo", cuyo procedimiento parte de aseveraciones en calidad de hipótesis y busca refutar o falsear tales hipótesis, deduciendo de ellas conclusiones que deben confrontarse con los hechos.

Considerando la profundidad estudio, el alcance de los resultados y la ubicación de los hechos en el tiempo, la investigación se fundamentó en tres tipos de estudios: descriptivo, explicativo y retrospectivo, dado que se centró en tratar de explicar por qué y cómo el gasto público que destinaron los gobiernos de países andinos a la educación llegó a generar una externalidad en el crecimiento económico, en el periodo 2000 -2015.

Para alcanzar el propósito planteado, se desarrolló un proceso de investigación bajo el enfoque cuantitativo. Se utilizó la técnica econométrica, es así que, el modelo econométrico utilizó datos de panel estableciendo para ello dos poblaciones de estudio: de corte transversal y temporal. Para la población transversal se seleccionaron a los países que conforman la región andina: Bolivia, Colombia, Ecuador y Perú y, para la población temporal se definió el periodo comprendido entre los años 2000 y 2015. Los criterios de selección de la población de corte transversal fueron: son países andino-amazónicos que comparten una biozona megadiversa, poseen un gran potencial de riqueza natural y la concentración de las estructuras productivas de estos países está basada principalmente en recursos minerales, energéticos y agrícolas; las mismas han caracterizado a sus economías desde tiempos coloniales. Por otro lado, en lo que respecta a la población temporal, se seleccionó el periodo 2000-2015, porque entre los años 2004 y 2014, existió un incremento exponencial de los precios internacionales de los recursos naturales, ocasionando que los países de la región andina expandan sus exportaciones de bienes primarios, situación que posibilitó la implementación de políticas sociales que mejoraron significativamente los indicadores sociales. Siguiendo a Mayorga (2000), Mahía (2000) y Park (2011), la ventaja de los modelos basados en datos de panel, comparado con los modelos de series de tiempo o de corte transversal es que el análisis con panel de datos permite la identificación de ciertos parámetros o preguntas sin la necesidad de hacer supuestos restrictivos. Así, por ejemplo, es posible ver cambios a nivel individual - que en este caso están representados por los países andinos - en el tiempo, es decir, brinda la posibilidad de observar las diferencias entre los países debidas a características propias, pero también circunstancias que hacen que estas características se 
modifiquen entre períodos.

La recolección de la información se realizó con el apoyo de las estadísticas e indicadores publicados por la Comisión Económica para América Latina y el Caribe (CEPAL) y por el Banco Mundial (BM). El procesamiento y análisis de la información se realizó utilizando el paquete estadístico STATA y diferentes pruebas estadísticas.

\section{RESULTADOS Y DISCUSIONES}

Los resultados de la investigación se presentan en tres puntos. En primer lugar, se describen las características propias o decisiones independientes que cada país andino realizó en cuento a la asignación del gasto público ${ }^{3}$; a continuación, se exponen las características comunes bajo las que se desarrollaron las políticas sociales en la región; finalmente, se explican los resultados obtenidos del modelo econométrico desarrollado.

\subsection{Asignación del gasto público en los países de la región andina}

Según información obtenida de las publicaciones realizadas por la CEPAL (2015), CEDLAS y BM (2017), CEDLAS y BM (2014), Atlas Resdal (s.f.) y Estadísticas CEPAL (s.f.) en el periodo 2010-2015, Bolivia asignó entre el 6.3\% y 7.6\% del PIB a educación, a continuación se encuentra Ecuador, con una asignación que oscila entre $4.3 \%$ y $5.2 \%$, en Colombia los valores fluctuaron entre $4.4 \%$ y $4.9 \%$, el país que menos recursos asignó fue Perú con valores entre $2.7 \%$ y $4.0 \%$ del PIB.

En relación a los recursos destinados a salud, en el periodo 2010-2014, Colombia asignó entre 6.6\% y 7.2\% del PIB, seguido por Ecuador, este país destinó entre 5.6\% y 9.2\% del PIB; en tercer lugar, se sitúa Bolivia con una asignación que oscila entre 5.4\% y 6.3\%, finalmente, Perú destinó entre $4.9 \%$ y $5.5 \%$ del PIB.

Situación similar a la presentada en Salud, ocurrió en la asignación de recursos a Defensa. Colombia destinó entre $3.1 \%$ y $3.6 \%$ del PIB, en segundo lugar, se encuentra Ecuador con una asignación entre $2.2 \%$ y $3.1 \%$, seguido por Bolivia, con valores que fluctúan entre $1.7 \%$ y $1.9 \%$ del PIB; el país que menos gasto público destino a Defensa fue Perú, con valores que se encuentran entre $1.2 \%$ y $1.7 \%$ del PIB.

\footnotetext{
${ }^{3}$ Hay dos clasificaciones ampliamente utilizadas que distinguen los componentes del gasto público: por su función y por características económicas. La primera se denomina clasificación funcional del gasto público y desglosa el gasto total en categorías como atención médica, educación y defensa. La segunda lleva el nombre de clasificación económica del gasto y divide el gasto total en gasto corriente y gasto de capital. Para efectos del presente trabajo, se toma en cuenta la clasificación funcional del gasto público (Armendariz y Carrasco, 2019).
} 
A modo de síntesis, los cuatro países presentaron una tendencia creciente en el gasto público como porcentaje del PIB, según se expone en la siguiente Tabla.

Tabla 1: Resumen de los recursos asignados como porcentaje del PIB

\begin{tabular}{|l|c|c|c|}
\hline \multicolumn{1}{|c|}{ País } & Educación & Salud & $\begin{array}{c}\text { Defensa } \\
\text { Nacional }\end{array}$ \\
\hline Bolivia & $6.3-7.6$ & $5.4-6.3$ & $1.7-1.9$ \\
\hline Colombia & $4.4-4.9$ & $6.6-7.2$ & $3.1-3.6$ \\
\hline Ecuador & $4.3-5.2$ & $5.6-9.2$ & $2.2-3.1$ \\
\hline Perú & $2.7-4.0$ & $4.9-5.5$ & $1.2-1.7$ \\
\hline
\end{tabular}

Fuente: Elaboración propia con datos extraídos de la CEPAL y del BM

\subsection{Características comunes bajo las que se desarrollaron las políticas sociales en la región andina}

Hacer un balance del contexto de 15 años en el que se desarrollaron las políticas sociales en la región andina es un trabajo bastante amplio y complejo, por lo tanto, simplemente se exponen aquellos que, desde un criterio personal, tuvieron efecto en la asignación del gasto público.

En el periodo 1982 y al menos 2004 las políticas económicas implementadas en América Latina y por ende en los países andinos estuvieron basadas bajo el llamado Consenso de Washington que respondía más a una lógica de estabilización que a una de desarrollo, donde la política social fue concebida como complementaria a la política económica, con objetivos muy poco vinculados (PNUD-BID, 1993).

El denominado Consenso de Washington fue diseñado inicialmente como un paquete de medidas ampliamente recomendadas por el BM y el FMI a todos los gobiernos de los países latinoamericanos, basado en: i) establecer una disciplina fiscal, ii) priorizar el gasto público en educación y salud, iii) llevar a cabo una reforma tributaria, iv) establecer tasas de interés positivas determinadas por el mercado, v) lograr tipos de cambio competitivos, iv) desarrollar políticas comerciales liberales, vii) promover una mayor apertura a la inversión extranjera, viii) privatizar las empresas públicas, ix) llevar a cabo una profunda desregulación y, x) garantizar la protección de la propiedad privada (Williamson, 1989).

Según la CEPAL (2004) las medidas implementadas no lograron reducir la pobreza; por el contrario, las brechas entre estratos socioeconómicos altos y bajos se ampliaron drásticamente en la mayoría de los países de la región. Ante tal situación, los países andinos realizaron una Reforma Social basada principalmente en la orientación, incremento de la eficiencia y descentralización del gasto público hacia el sector social, focalizada en los grupos de personas con mayores carencias y concentrada principalmente en educación, saneamiento y salud (Medellin, 2004). 
La descentralización estuvo básicamente ligada a dotar responsabilidad sobre la provisión de servicios sociales, estableciendo sistemas de transferencias desde el gobierno central hacia los municipios; sistemas que incluían criterios de compensación que permitían nivelar la capacidad desigual de los gobiernos locales para recaudar. El proceso de descentralización empezó en Colombia con la Constitución de 1991 (Ley N ${ }^{\circ}$ 1381), en Ecuador mediante la Ley de Modernización del Estado de 1993 (Ley No 50), en Bolivia en 1994, mediante la Ley de Participación Popular (Ley No 1551) y, por último, en Perú a partir de la Reforma Constitucional de 2002 (Ley N 27680) (Barrios, 2017).

En el año 2000, se realizó la Cumbre del Milenio de las Naciones Unidas, 189 países entre ellos los países de la región andina firmaron la llamada Declaración del Milenio, comprometiéndose a realizar los esfuerzos necesarios para la consecución de los objetivos denominados “Objetivos de Desarrollo del Milenio” y las metas allí planteadas, resaltándose como ejes centrales la reducción de la pobreza, el desarrollo sostenible, la igualdad de género y el respeto a los derechos humanos. En consecuencia, la firma de este acuerdo internacional ha impulsado un importante número de iniciativas llevadas a cabo por los países andinos tanto a nivel de legislación, como de política social, dirigidas a la ampliación de la cobertura de servicios sociales básicos en educación, salud, agua y saneamiento (Naciones Unidas, 2000).

Adicionalmente, se puede señalar que en el año 2001 la Comunidad Andina, dispuso la elaboración de un Plan Integrado de Desarrollo Social (PIDS) con el propósito de promover la cooperación y coordinación de políticas y acciones entre los países miembros, así como la ejecución de proyectos de alcance regional, con el fin de agregar valor a las políticas nacionales contra la pobreza, la exclusión y la desigualdad social en el marco de los compromisos asumidos en la Cumbre Mundial sobre Desarrollo Social de 1995 y los Objetivos de Desarrollo del Milenio, el mismo fue aprobado mediante Decisión 601 en septiembre de 2004. El PIDS se basó en tres líneas de trabajo: la cooperación técnica en políticas sociales entre los Estados miembros de la Comunidad Andina; el seguimiento y evaluación conjuntos de objetivos y metas sociales compartidos y; la ejecución de programas y proyectos comunitarios de alcance regional referidos a una amplia gama temática, relacionados con: salud, educación, interculturalidad, desarrollo rural, sociolaborales, seguridad alimentaria, aprovechamiento sostenible de la biodiversidad, preservación y gestión de fuentes compartidas del agua y desarrollo social en zonas de frontera (Comunidad Andina, 2013).

Otro aspecto por mencionar es que, a partir de 2004, la región andina retoma un crecimiento económico relativamente sostenido, basado principalmente en la expansión de sus exportaciones de bienes primarios, como ser: cobre en Perú, petróleo en Ecuador, carbón en Colombia y 
minerales e hidrocarburos en Bolivia, resultado del acelerado crecimiento de la economía china que impulsó la demanda de minerales y alimentos. La bonanza económica ocasionó un aumento significativo de la inversión pública en educación, salud y seguridad social y, en consecuencia, los países andinos tendieron a efectuar políticas sociales con un mayor enfoque universal basadas en la noción de derechos y en la consolidación de Programas de Transferencias Monetarias Condicionadas. Bajo ese escenario económico alentador, los países de la región andina adoptaron una nueva estrategia de desarrollo social, que en palabras de Maurizio (2010) estuvo basada en cuatro ejes principales: expansión del gasto en educación y salud, y de la seguridad social en general; aumento en los salarios medios reales y en los salarios mínimos; incremento en la cobertura y calidad de la protección social a los trabajadores y expansión y consolidación de transferencias condicionadas de ingresos a los hogares pobres. Respecto a las transferencias condicionadas, los cuatro países que conforman la región andina implementaron Programas de Transferencias Condicionadas destinados a reducir la deserción escolar, la mortalidad infantil, malnutrición y desnutrición. Colombia dio inicio en al año 2001, con el Programa "Más Familias en Acción”, años más tarde, en el 2007, implementó el Programa "Red Unidos”. En el año 2004, Ecuador, inicia el Programa de Transferencias Condicionadas con el "Bono de Desarrollo Humano” y en el año 2011 el Programa “Desnutrición Cero”. Perú en el año 2005, con el Programa de Apoyo Directo a los más Pobres denominado "Juntos". Finalmente, en el año 2006, Bolivia inicia el Programa de Transferencias Condicionadas denominado "Bono Juancito Pinto" y en el año 2009 implementó el “Bono Madre Niño-Niña” y el "Bono Juana Azurduy de Padilla".

Adicionalmente, en la Cumbre Mundial sobre Desarrollo Social que se realizó en el año 2005, los gobiernos se comprometieron a desarrollar Estrategias Nacionales de Desarrollo más ambiciosas, para construir países que sean socialmente incluyentes, que generen empleo, crecimiento económico y estabilidad política. Asimismo, desarrollar fuentes innovadoras de financiación, incluidas acciones por grupos de países para implementar el mecanismo internacional de financiación y otras iniciativas para financiar proyectos de desarrollo, en particular en el sector de la salud (Naciones Unidas, 2005).

A modo de síntesis, en el periodo de estudio (2000-2015), los países de la región andina, se desarrollaron bajo contextos, procesos y políticas similares que permiten hablar de una posible reacción conjunta (sistémica) en cuanto a los resultados de las políticas sociales y de la dotación de los bienes públicos. 


\subsection{Resultados del modelo econométrico}

El modelo econométrico se basó en las variables que propone el modelo de crecimiento endógeno propuesto por Barro (1990):

$$
Y=A K^{\alpha} G^{1-\alpha}
$$

Donde; $K$ representa el capital, tanto físico como humano, $A$ la tecnología y $G$ los bienes públicos. La incorporación de los bienes públicos responde al argumento de que la inversión del Estado en infraestructura, investigación, educación y servicios públicos produce externalidades que contribuyen al crecimiento de la productividad de los sectores productivos creando de esta manera un vínculo potencialmente positivo entre la provisión de bienes públicos y el crecimiento económico.

Bajo la anterior consideración y siguiendo la propuesta de Greene (2008) y Gujarati (2010), se especificó el siguiente modelo econométrico:

$$
Y_{i t}=\alpha+\beta_{1} X_{1 i t}+e_{i t}
$$

Donde:

i: son los países sujetos a estudio: Bolivia, Colombia, Ecuador y Perú

t: el periodo de análisis: 2000, 2001, 2002, .., 2015

$Y_{i t}$ : la variable dependiente (crecimiento económico) del país $i$ en el año $t$.

$X_{i t}$ : son las variables explicativas (inversión pública, representada por la formación bruta de capital fijo y los bienes públicos, desagregados en: educación, salud, vivienda, seguridad social y gasto militar) del país $i$ en el año $t$.

$e_{i t}:$ es el término de error.

El resultado del proceso de elaboración del modelo econométrico determinó que el modelo econométrico basado en datos de panel que mejor se ajusta para explicar la relación entre el crecimiento económico y la asignación del gasto público a los bienes públicos de los países que conforman la región andina, en el periodo de estudio, corresponde a un modelo dinámico de datos de panel de efectos fijos, que se presenta a continuación: ${ }^{4}$

$$
\text { (1.3.) } C E_{i t}=2.77+0.16 C E_{i t-1}+1.3 E D_{i t-1}+0.56 D E_{i t-1}-1.1 S A_{i t}+0.21 F B C_{i t}
$$

Donde: $C E$ representa el crecimiento económico de cada país de la región andina en el periodo 2000-2015; ED, $D E$, $S A$ son las variables asignadas a los recursos que los gobiernos destinaron al gasto público: educación, defensa y salud como porcentaje del PIB, de cada país

\footnotetext{
${ }^{4}$ Para determinar el modelo econométrico que mejor se ajusta al objeto de estudio, es necesario realizar pruebas de especificación y de validez, que en muchos casos requiere la incorporación o eliminación de variables que no resultar ser estadísticamente significativas. El modelo econométrico resultante se encuentra en Anexos.
} 
andino en el periodo 2000-2015, y FBC representa a la inversión pública definida como la Formación Bruta de Capital Fijo también medida en porcentaje del PIB de los países que conforman la región andina; esta variable incluye infraestructura física financiada por inversión pública, como son: carreteras, sistemas de suministro de agua y alcantarillado, redes de distribución de gas y electricidad, escuelas, hospitales, entre otros. En ese escenario, los resultados señalan:

Primero: el hecho de que el modelo econométrico resultante corresponda a un modelo de efectos fijos, significa que la dotación de los bienes públicos afecta por igual a todos los países de la región andina y que las diferencias entre ellos se deben a las características o decisiones propias de cada país, permite hablar de una reacción conjunta o sistémica del impacto del gasto público y de la inversión pública en el crecimiento económico de la región andina. El hecho de que el modelo de efectos fijos haya sido el modelo seleccionado confirma lo expuesto por Franco, Ramos y Hernández (2016:301) "si se trabaja con una muestra seleccionada a conveniencia y cuando el número de sujetos o periodo de tiempo no sea grande, se recomienda el modelo de efectos fijos".

Segundo: el signo positivo de los coeficientes señala que el gasto público destinado a "Educación” y a "Defensa Nacional” generó una oportunidad para el crecimiento económico en los países andinos. Resultado que coincide con los postulados de Lucas (1988) y de Howitt (2004), quienes como producto de las investigaciones realizadas concluyen que una fuerza laboral educada tiene mayor capacidad de innovación y competitividad para desempeñar actividades productivas, ya sea para crear, ejecutar o adaptar nuevas tecnologías, por lo tanto, mejora la productividad de los trabajadores; situación que llega ocasionar un mayor crecimiento económico. De la misma manera, los resultados apoyan los resultados de los trabajos de Benoit (1973), Biswas-Ram (1986), Atahuichi (2002) y Crespo-Reitschuler (2003), que concluyen señalando que la necesidad de invertir en defensa se deriva de la necesidad de garantizar un ambiente de normalidad y tranquilidad que un país requiere para el desenvolvimiento de sus actividades, generando de esta manera externalidades positivas en la productividad del país y por ende un mayor crecimiento económico.

Por su parte, el signo negativo del coeficiente del gasto público destinado a "Salud" evidencia que los recursos asignados a este bien público no llegaron a constituirse en una oportunidad para el crecimiento económico. Al respecto, si se toma en cuenta que en promedio los países de la región andina destinaron más recursos como porcentaje del PIB a "Salud" que a "Educación", pone en evidencia que, en el periodo de estudio, el gasto público en salud no necesariamente se relacionó con mayor eficiencia del gasto público. La anterior afirmación se respalda en la publicación editada por Izquierdo, Pessino y Vueltin (2018) donde se expone que 
en el sector salud, la ineficiencia del gasto puede darse cuando los pacientes no reciben la mejor atención posible para un determinado nivel de recursos asignados (ineficiencia asignativa) o cuando el consumo excesivo de recursos roba a otros pacientes la posibilidad de tratamiento y ganancias en salud (ineficiencia técnica). La publicación, presenta evidencia empírica de los niveles de eficiencia de los sistemas de salud basada en el análisis envolvente de datos; con información del periodo 2006-2015; el desempeño de la eficiencia se mide utilizando tres categorías: salud, acceso a los servicios y equidad en el acceso a los servicios ${ }^{5}$. Los resultados a nivel de los países andinos muestran que: Colombia obtuvo una puntuación de 0.938 , seguido por Ecuador con 0.916, en tercer lugar, se ubica Perú con 0.887 y, finalmente se encuentra Bolivia con 0.845. Adicionalmente, las puntuaciones sitúan a Colombia en la mitad inferior de las puntuaciones de 60 de los países que formaron parte del estudio, y a Ecuador, Perú y Bolivia en el $25 \%$ inferior.

Tercero: los valores de los coeficientes permitieron cuantificar el impacto del gasto público en el crecimiento económico. Al respecto:

- El incremento de 1 p.p. del gasto público destinado a "Defensa Nacional" ocasionó que el crecimiento económico incrementara en 0.56 p.p., pero el incremento ocurrió un año después de la asignación de los recursos.

- El incremento de 1 p.p. en el gasto público asignado a "Educación” generó un incremento de 1.3 p.p. en el crecimiento económico un año después que los gobiernos incrementaron los recursos.

- El incremento de 1 p.p. en los recursos destinados a "Salud" ocasionó una disminución de 1.1 p.p. en el crecimiento económico, esta disminución se dio el mismo año que los gobiernos destinaron los recursos.

Cuarto: al ser el coeficiente de la variable "Educación" positivo y el mayor en comparación al resto de las variables, evidencia que el gasto público destinado a Educación fue el que mayor impacto tuvo en el crecimiento económico de los países de la región andina, por lo tanto, las políticas sociales dirigidas a mejorar la educación de los habitantes de los países de la región andina llegaron a constituirse en una oportunidad para el crecimiento.

\footnotetext{
${ }^{5}$ El desempeño de la eficiencia se mide para ocho productos del sistema de salud agrupados en tres categorías: i) salud: esperanza de vida al nacer y a los 60 años, mortalidad de menores de 5 años y años de vida ajustados por discapacidad; ii) acceso a los servicios: tasas de inmunización DTP (Difteria, Tétanos y Tos ferina) y de atención especializada durante el parto; iii) equidad en el acceso a los servicios: tasas de atención especializada durante el parto rural vs. urbano y más pobres vs. más ricos.
} 
Quinto: existen otras variables que impactaron positivamente en el crecimiento económico de los países, entre las que se encuentran:

- La Formación Bruta de Capital (FBC), señala que un incremento de 1 p.p. en la FBC causó que el crecimiento económico de la región andina aumente en 0.21 p.p. en el mismo año que se dio el incremento en la FBC. Resultado que apoya el trabajo de Manuelito y Jiménez (2013), quienes señalan que la inversión adecuadamente canalizada es un elemento fundamental para el desarrollo económico, en el corto y mediano plazo la inversión genera crecimiento mediante el impulso a la demanda agregada; adicionalmente, en el largo plazo, la inversión de calidad genera cambios tecnológicos, permite desarrollar una mayor capacidad productiva y fomenta la reasignación de recursos hacia los sectores más productivos.

- El crecimiento económico también tiene un efecto positivo en el crecimiento del siguiente año, el impacto se da en 0.16 p.p.

Integrando los resultados, el proceso de investigación realizado permitió identificar que en el periodo de estudio (2000-2015), los países de la región andina, se desarrollaron bajo contextos y políticas similares, donde las características y decisiones propias de cada país inherentes a los procesos comunes, si bien los diferenciaron, a su vez permiten hablar de una reacción conjunta a nivel de la región, en cuanto a los resultados de las políticas sociales y de la asignación del gasto público. Análisis contrastado con los resultados del modelo econométrico de efectos fijos.

La reacción conjunta o sistémica permitió distinguir que el gasto público destinado a "Educación” y a "Defensa" generó una oportunidad en el crecimiento económico. Adicionalmente, se identificó que el gasto público asignado a "Educación" es el que más aporta o contribuye en el crecimiento económico. La naturaleza del vínculo entre educación y crecimiento económico depende de un conjunto de factores como: el tipo de políticas sociales implementadas, las características del mercado laboral, entre otros. Una forma de analizar la dependencia es: "si un país dirige acciones de política social para lograr una mejor educación, pero existen escasas posibilidades de un empleo formal, a pesar que la población cuente con mayores capacidades no podrá ingresar al mercado laboral y por ende no se logrará el efecto esperado en el crecimiento económico".

En ese orden de ideas, si bien es necesario que las acciones de la política social estén orientadas a reducir los niveles de marginación del sistema escolar y a mejorar la cobertura y acceso educativo, las tasas de retención y promoción, los niveles de rendimiento, la calificación de la mano de obra, entre otros, es igualmente necesario que las políticas sociales vayan acompañadas de políticas de desarrollo productivo y de políticas laborales. En el primer caso, 
políticas de fomento a las micro, pequeñas y medianas empresas, siendo que estas, no solo aportan al crecimiento económico sino adicionalmente son generadoras de empleo formal y, en el segundo, políticas que mejoren la inserción y el acceso a oportunidades laborales.

Otra forma de fortalecer la relación o vínculo de la educación con el crecimiento económico es a través de la implementación de políticas y programas de desarrollo múltiple, como son aquellos que vinculan el apoyo al sector productivo con la formación y capacitación.

Los resultados también identificaron que el gasto público destinado a "Salud" no logró generar una oportunidad en el crecimiento económico en el periodo de estudio. Esta situación, no significa que se deban dejar de implementar políticas sociales dirigidas a disminuir la morbimortalidad, mejorar la nutrición, el acceso a la salud, entre otros, primero, porque es un derecho humano fundamental y segundo, porque genera externalidades positivas en la educación, como se expresa líneas arriba, una población sana incrementa y hace más efectivos los años de escolaridad posibilitando una mayor capacitación y, adicionalmente mejora la productividad de los trabajadores, por ende, logra impulsar un crecimiento económico incluyente.

Los puntos expuestos, visualizan la importancia de articular de manera interdependiente a las políticas económicas y sociales como condicionantes para un crecimiento con inclusión y equidad.

Un punto que amerita ser aclarado es que debido a que el gasto público está a cargo del Estado, surge la necesidad de analizar la eficiencia del gasto público desagregado en: i) eficiencia técnica, que analiza cuál es la relación entre recursos y resultados, dada la distribución del gasto actual; ii) eficiencia asignativa, relacionado con la priorización del gasto en las partidas con mayores retornos socioeconómicos; es decir, a como los gobiernos asignan su gasto en diferentes funciones, como son: educación, salud, inversión y defensa; con el fin de maximizar la productividad y el crecimiento de la economía y, iii) eficiencia de la gestión, se refiere a la calidad de los procesos e instituciones que administran el gasto público. Sin embargo, a la fecha existen pocas bases de datos del gasto total en inversión pública desagregado bajo las clasificaciones cruzadas económica-institucional-funcional (Armendariz y Carrasco, 2019).

\section{CONCLUSIONES}

Como producto del trabajo, se determinó que el gasto público destinado a la educación generó una externalidad positiva en el crecimiento económico de los países de la región andina en el periodo 2000-2015, aceptando de esa manera la hipótesis planteada como respuesta al trabajo de investigación realizado. 
Adicional a lo anterior, la evidencia empírica y los resultados del modelo econométrico permiten indicar que las decisiones propias de cada país derivaron de los procesos comunes que estuvieron presentes en la región, lo que permitió hablar de una reacción sistémica a nivel regional en cuanto a los resultados de la asignación del gasto público.

La reacción conjunta (sistémica) en el crecimiento económico ante cambios en la asignación del gasto público permitió determinar, a través del signo positivo del coeficiente de las variables "Educación y "Defensa", que el gasto público asignado estos tipos de bienes llegaron a generar una externalidad en el crecimiento económico de la región andina. Por su parte, el signo negativo del estimador de la variable "Salud" reveló que el gasto público asignado a la salud no llegó a generar una oportunidad en el crecimiento económico. Finalmente, se tiene que el gasto público destinado a "Educación" fue e gasto que generó mayores oportunidades en el crecimiento económico de los países de la región andina. Por todo lo expuesto, el trabajo de investigación llegó a cumplir con el propósito planteado.

Finalmente, es necesario señalar que el ámbito de estudio del crecimiento económico es bastante amplio, lo que causó qua durante el desarrollo del trabajo surgieran diferentes inquietudes, las mismas quedan planteadas como recomendaciones para futuras investigaciones. Por lo tanto, se recomienda realizar estudios que: i) profundicen el estudio de las políticas sociales incorporando en el análisis los factores de poder, las tensiones sociales y los aspectos culturales que inciden en el éxito de las políticas y en el crecimiento económico; ii) exploren la relación entre el gobernabilidad, crecimiento económico y desarrollo, a partir de un análisis de las características de las políticas sociales; iii) sistematicen evidencias empíricas sobre los impactos y resultados de las políticas sociales en el crecimiento económico; iv) examinen las políticas sociales dirigidas a la dotación de bienes públicos desde un enfoque de igualdad, en cuanto a disparidades género, étnico-culturales y generacionales, v) analicen la eficiencia del gasto público, según su función, desagregado en eficiencia técnica y eficiencia asignativa, siendo que ambos tipos de eficiencia resultan cruciales para promover el crecimiento económico a largo plazo y mejorar la equidad, y vi) incluyan en el debate los fracasos de los modelos de desarrollo y de políticas sociales desde una visión que da las disciplinas como la ciencia política y la sociología.

\section{REFERENCIAS BIBLIOGRÁFICAS}

Acemoglu, D., y otros. (2004). Institutions as the fundamental cause of long-run growth. National Bureau of Economics Research. WP 10481.

Aghion, P., y Howit P. (1998). Endogenous Growth Theory. Massachusetts Institute Technology.

Armendariz, E., y Carrasco, H. (2019). El gasto en inversión pública de América Latina. Cuánto, quién y en qué. Banco Interamericano de Desarrollo. División de Gestión Fiscal. Documento para Discusión No. IDB-DP-697. 
Atahuichi, D. (2002). Estudio y Análisis de los Desequilibrios Financieros del Gasto Público Militar en Bolivia en la Década del 90. En: Research and Education in Defense and Security Studies.

Barrios F. X. (2017). Las recientes reformas de regionalización en Colombia, Perú, Ecuador y Bolivia. Hacia un marco conceptual, Geopolítica(s). Revista de estudios sobre espacio y poder, 8(1). 51-89.

Barro, R. (1990). Economic Growth in a Cross Section of Countries. En: Quarterly Journal of Economics, Cambridge Center, Cambridge, MA., U.S.A.

Barro, R. (1997), Determinants of Economic Growth. Cambridge: MIT Press. U.S.A.

Becker, G. (1995). Human Capital and Economic Growth. Prague Economics Papers 4.

Benoit, E. (1973). Defense and Economic Growth in Developing Countries. Lexington. Massachusetts. Lexington Books.

Biswas, B. y Ram, R. (1986). Military Expenditures and Economic Growth in Less Developed Countries: An Augmented Model and Further Evidence. Economic Development and Cultural Change. University of Chicago Press. Vol 34(2).

Comunidad Andina. (2013). Informe de Gestión mayo 2010-febrero 2013.

CEDLAS y BM. (2017). Base Socio-económica para Latinoamérica y el Caribe [Base de datos]. Recuperado de http://www.cedlas.econo.unlp.edu.ar/

CEDLAS y BM. (2014). A Guide to SEDLAC: Socio-Economic Database for Latin America and the Caribbean. Boards.

CEPAL. (2004). Desarrollo de infraestructura y crecimiento económico: revisión conceptual. Santiago.

CEPAL. (2015). Educación, cambio estructural y crecimiento inclusivo en: América Latina. Santiago. Levy. Santiago y Norbert Schady. 2013.

CEPAL. (2016). Estadísticas e indicadores. Cepal-cepalstat [Base de datos]. Recuperado de: http://estadisticas.cepal.org/cepalstat/

Crespo, J. y Reitschuler, G. (2003). Guns or butter? Revisited: Robustness and Nonlinearity Issues in Defense-Growth Nexus. Working paper No. 0310. Department of Economics. University of Vienna.

Dick, S. (2010). Profitof Education. Praeger.

Franco, M. y otros. (2016). Combinación de datos de corte transversal y series temporales. Universidad del Oriente

Glaeser, E. y otros. (2004). Do institutions cause growth?. Journal of Economic Growth 9 (3). 271-303.

Greene, W. H. (2008). Econometric Analysis. Sexta ed. Upper Saddle River. NJ: Prentice Hall.

Gujarati, D., Porter, D. (2010). Econometría, Quinta ed. México DF: McGraw Hill.

Izquierdo, A., y otros. (ed.). (2018). Mejor gasto para mejores vidas. Cómo América latina y el Caribe puede hacer más con menos. Banco Interamericano de Desarrollo. Recuperado de: www.iadb.org/DIA2018gasto 
Kaufmann, D., y Kraay, A. (2002). Growth Without Governance. The World Bank World Bank Institute and Development Research Group. Paper 2928.

Lucas, R. (1988). On the mechanics of economic development. Journal of Monetary Economics No. 21. 3-42.

Mahía, R. (2000). Introducción a la Especificación y Estimación de Modelos con Datos de Panel. Doctorado I.L.R. KLEIN.

Mayorga, M., y Muñoz, E. (2000). La Técnica de Datos de Panel. Una guía para su uso e interpretación. Banco Central de Costa Rica. Departamento de Investigaciones Económicas.

Mankiw, G., otros. (1992). A Contribution to the Empirics of Economic Growrh. The Quarterly Journal of Economics No.107.

Medellin, P. (2004). Política y políticas públicas en los procesos de reformas en América Latina. Similitudes y Diversidades. CEPAL.

Manuelito, S., y Jiménez, L. F. (2013). Rasgos estilizados de la relación entre inversión y crecimiento en América Latina y el Caribe, 1980-2012. Serie Macroeconomía del Desarrollo. Comisión Económica para América Latina y el Caribe.

Maurizio, R. (2010). The role of social policy in eradicating poverty: the Latin Americanexperience. Buenos Aires: CONICET - Universidad General Sarmiento.

Mogollón, A. (2009). Reflexiones en salud pública. Bogotá.

Naciones Unidas. (2000). Resolución aprobada por la Asamblea General 55/2. Declaración del Milenio

Naciones Unidas. (2005). La cumbre mundial de 2005, Reunión plenaria de alto nivel, 15-16 de septiembre de 2005.

Park, H. M. (2011). Practical Guides to Panel Data Modeling: A Step-by-step Analysis Using Stata. Tutorial Working Paper. Graduate School of International.

PNUD-BID. (1993). Reforma Social y Pobreza.

Rajan. R., Zingales. G. (2006). The Persistence of Underdevelopment: Institutions, Human Capital, or Constituencies? NBER Working Paper No. 12093. Issued in March 2006.

Ravallion, M. (2014). Income inequality in the developing world. Science. 344(6186). 851-855.

Romer, P. (1986). Increasing Returns and Long-Run Growth. Journal of Political Economy. No. 94.

Romer, P. (1990). Endogenous Technological Change. Journal of Political Economy No. 98. 71102.

Sala-i-Martin, X. (2002). Apuntes de crecimiento económico. Segunda edición.

Solow, R. (1957). Technical Change and the Aggregate Production Function. The MIT Press. USA.

Valdés, J., Espina, M. (2011). América Latina y el Caribe: La política social en el nuevo contexto - Enfoques y experiencias. UNESCO. Montevideo. 
Williamson, J. (1989). What Washington Means by Policy Reform. En Williams, Jhon (ed): Latin American Readjustment: How Much has Happened. Washington. Institute for International Economics 1989.

[Atlas Resdal,s.f.]. Recuperado de: http://atlas.resdal.org/

[Estadisticas CEPAL]. Recuperado de: http://estadisticas.cepal.org/

\section{ANEXOS}

\section{Resulting econometric model}

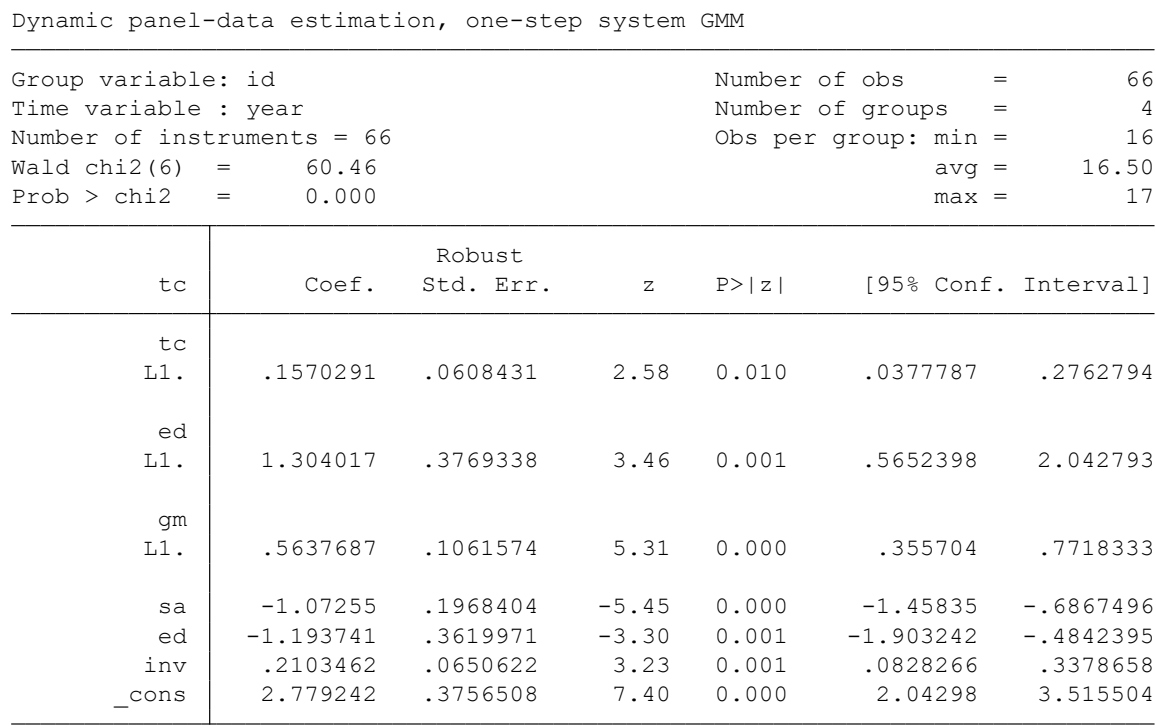

\begin{tabular}{l} 
Arellano-Bond test for $\operatorname{AR}(1)$ in first differences: $z=-1.58$ Pr $>z=0.114$ \\
Arellano-Bond test for $\operatorname{AR}(2)$ in first differences: $z=0.44 \operatorname{Pr}>z=0.660$ \\
\hline Sargan test of overid. restrictions: chi2(59) $=61.30$ Prob $>$ chi2 $=0.394$
\end{tabular}

\section{Condition 1: Validity of instruments}

Ho: Overidentification restrictions are valid.

Ha: Overidentification restrictions are invalid.

The Sargan test shows that the value of the probability (0.394) is greater than 0.05 , therefore, the instruments used in the estimation are valid and there is no overidentification.

\section{Condition 2: Autocorrelation}

Ho: there is no autocorrelation.

Ha. There is autocorrelation

The Arellano and Bond Test provides that the $\mathrm{pr}>\mathrm{z}=0.66$ for $\mathrm{Ar}(2)$, since the value is greater than 0.05 , the null hypothesis is not rejected, concluding that there is no autocorrelation

\section{ANEXOS}

\section{Modelo econométrico resultante}




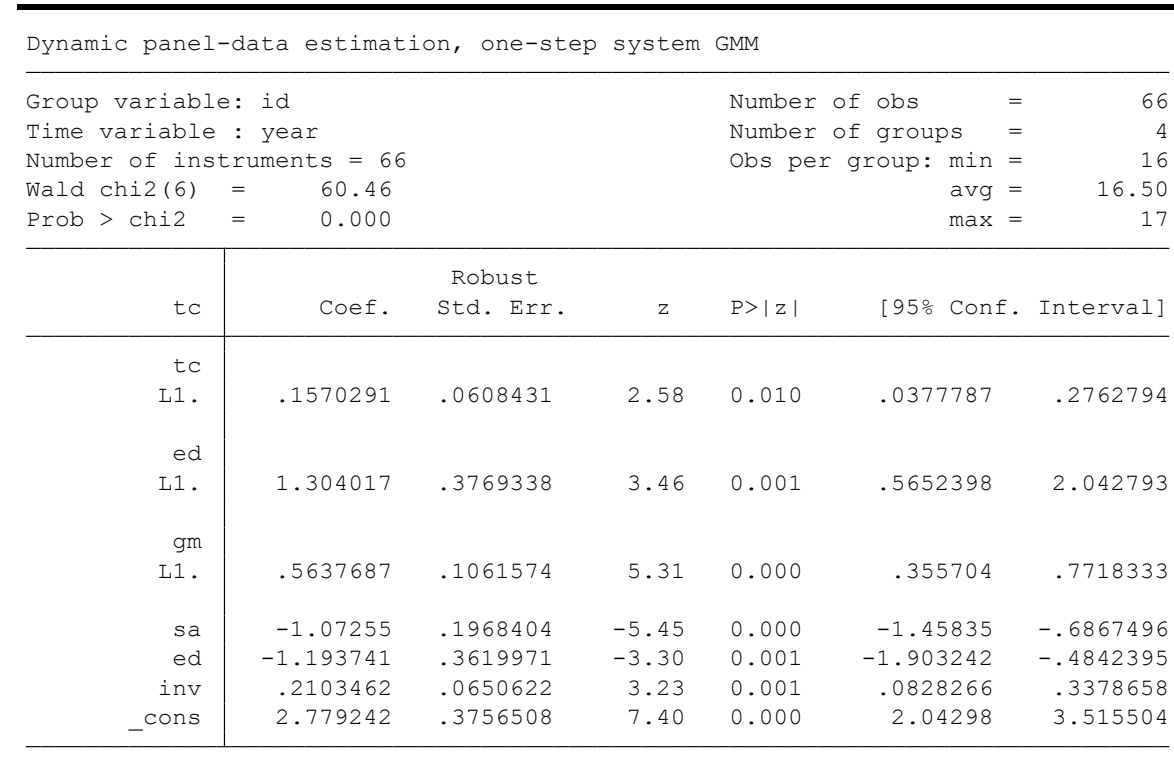

Arellano-Bond test for AR(1) in first differences: $z=-1.58$ Pr $>z=0.114$ Arellano-Bond test for AR(2) in first differences: $z=0.44 \operatorname{Pr}>z=0.660$

Sargan test of overid. restrictions: chi2(59) $=61.30$ Prob $>$ chi2 $=0.394$

\section{Condición 1: Validez de los instrumentos}

Ho: Las restricciones de sobreidentificación son válidas.

Ha: Las restricciones de sobreidentificación no son válidas.

El test de Sargan muestra que el valor de la probabilidad (0.394) es mayor a 0.05 , por lo tanto, los instrumentos utilizados en la estimación son válidos y no existe sobreidentificación.

\section{Condición 2: Autocorrelación}

Ho: no existe autocorrelación.

Ha. Existe autocorrelación

El Test de Arellano y Bond proporciona que la $\mathrm{pr}>\mathrm{z}=0.66$ para $\operatorname{Ar}(2)$, al ser el valor mayor de 0.05 no se rechaza la hipótesis nula, concluyendo que no existe autocorrelación

\section{Conflicto de intereses / Competing interests:}

La autora declara que no incurre en conflictos de intereses.

\section{Rol de los autores / Authors Roles:}

No aplica.

\section{Fuentes de financiamiento / Funding:}

La autora declara que no recibió un fondo específico para esta investigación.

\section{Aspectos éticos / legales; Ethics / legals:}

La autora declara no haber incurrido en aspectos antiéticos, ni haber omitido aspectos legales en la realización de la investigación. 